\title{
Analysis of Outage Probability and Throughput for Energy Harvesting Full-Duplex Decode-and-Forward Vehicle-to-Vehicle Relay System
}

\author{
Ba Cao Nguyen $\mathbb{D}^{1},{ }^{1}$ Nguyen Nhu Thang, ${ }^{1}$ Tran Manh Hoang, ${ }^{1}$ and Le The Dung $\mathbb{D}^{2,3}$ \\ ${ }^{1}$ Telecommunications University, Khanh Hoa Province, Vietnam \\ ${ }^{2}$ Division of Computational Physics, Institute for Computational Science, Ton Duc Thang University, Ho Chi Minh City, Vietnam \\ ${ }^{3}$ Faculty of Electrical and Electronics Engineering, Ton Duc Thang University, Ho Chi Minh City, Vietnam
}

Correspondence should be addressed to Le The Dung; lethedung@tdtu.edu.vn

Received 5 January 2020; Revised 15 April 2020; Accepted 8 May 2020; Published 29 May 2020

Academic Editor: Gianluigi Ferrari

Copyright ( $2020 \mathrm{Ba}$ Cao Nguyen et al. This is an open access article distributed under the Creative Commons Attribution License, which permits unrestricted use, distribution, and reproduction in any medium, provided the original work is properly cited.

\begin{abstract}
In this paper, we evaluate the performance of a vehicle-to-vehicle (V2V) system where full-duplex relay (FDR) harvests the energy from source and uses decode-and-forward (DF) protocol to forward data from source to destination. Unlike existing works about FDR systems, we consider the scenario that both relay and destination are moving vehicles, leading to the channel between relay and destination characterized by double (cascade) Rayleigh fading. We successfully obtain the closed-form mathematical expressions of the outage probability (OP) and throughput of the considered energy harvesting- (EH-) FDR-V2V system. Based on these expressions, the system performance is investigated through various scenarios. Numerical results indicate that the performance of the considered system is reduced compared with that of the system over Rayleigh fading channels. We also observe that there is an optimal EH time duration that minimizes the OP and maximizes the throughput. This value depends on the transmission power of source. Furthermore, the OP goes to outage floor faster due to the impact of the residual selfinterference (RSI), especially when RSI is high. All analysis results are verified by Monte-Carlo simulations.
\end{abstract}

\section{Introduction}

Recently, energy harvesting (EH) has attracted great attention due to the advantages and applications of this technique in wireless sensors, microcontrollers, and displays [1-4]. Compared with the traditional wireline power supply, the wireless power supply using EH can be applied for the devices whose batteries cannot be charged by traditional methods, such as the devices in body area networks. Therefore, harvesting energy from the environment is a promising approach to prolong the lifetime of energy-constrained wireless networks. Among other renewable energy sources such as solar and wind, background radio-frequency (RF) signals radiated by ambient transmitters can be a viable new source for wireless power transfer (WPT). On the other hand, RF signals have been widely used as a vehicle for wireless information transmission (WIT). Simultaneous wireless information and power transfer (SWIPT) becomes appealing since it realizes both useful utilizations of RF signals at the same time and thus potentially offers great convenience to mobile users.

Meanwhile, full-duplex (FD) communications can increase the spectral efficiency two times compared with the traditional half-duplex (HD) communications because FD devices simultaneously transmit and receive signal at the same time and on the same frequency band. Thus, the FD technique is able to satisfy the requirement of the future wireless network such as the fifth generation (5G) and beyond [5-8]. However, the residual self-interference (RSI) after selfinterference cancellation (SIC) increases the outage and bit error rate (BER) and reduces the capacity of FD communication systems. Fortunately, the researches and measurements have demonstrated that the FD systems can be deployed in realistic scenarios with the help of the recent advantage techniques such as antenna design, analog, and digital signal processing. By using three domains for cancellation such as antenna domain suppression, analog, and digital domain 
cancellation, the self-interference (SI) can be suppressed up to $110 \mathrm{~dB}$, making the RSI as small as the noise floor [9, 10]. Nowadays, the FD transmission is exploited at relay to enhance the coverage and reliability of the wireless communication systems $[6,11-14]$. It is demonstrated that by using FD relay (FDR), the wireless systems can get higher capacity with small performance loss compared with the traditional HD relay (HDR) wireless systems.

To prolong the lifetime of wireless devices in FDR communication systems, the $\mathrm{EH}$ technique has been considered in many research works in the literature [3, 12, 15-19]. Based on mathematical analysis, the performance of EH-FDR systems has been investigated in various scenarios such as over Nakagami- $m$ fading channel [12], Rayleigh fading [3, 1518], and $\alpha-\mu$ environment [19]. These works derived the analysis expressions of the outage probability (OP) $[3,12$, 15-19], symbol error probability (SEP) [12, 15], and ergodic capacity $[16,18]$ of the EH-FDR systems in the case that only FD relay harvests the energy from source or both source and FD relay harvest the energy from power beacon (PB). Numerical results indicated that these systems can operate in practical scenarios with high transmission power of source or PB. Moreover, using PB with multiple antennas can significantly increase the harvested energy at source and FD relay, thus improving the OP and SEP performance. On the other hand, the RSI due to FD transmission mode has a great impact on the performance of EH-FDR systems. It makes the OP and SEP of the EH-FDR systems go to error floor in high signalto-noise ratio (SNR) regime. In addition, choosing a suitable $\mathrm{EH}$ time duration is very important because that value can minimize the OP and SEP of the EH-FDR systems.

Additionally, various works in the literature have proposed several architectures for SWIPT receivers such as ideal receiver, time switching (TS) receiver, and power splitting (PS) receiver $[20,21]$. The advantages and disadvantages of these SWIPT receivers have been discussed. Specifically, the ideal receiver is not suitable for practical circuits, due to the fact that the receiver could not be able to decode information and harvest energy from the same signal [21]. Meanwhile, the TS and PS receivers are applicable in practical. In TS protocol, the receiver harvests energy and retrieves information in two time slots. In PS protocol, the receiver splits the received signal into two streams, one for $\mathrm{EH}$ and the other for information decoder [21].

Today, vehicle-to-vehicle (V2V) communication systems are fast developed due to their crucial role in the road safety applications, especially in the cooperative driving and semiautonomous driving [22]. In that context, FDR is used to reduce the transmission delay between vehicles in intelligent transportation systems (ITS) [22-24]. In the literature, various works have investigated the performance of FDR-V2V systems via the mathematical expressions of OP and SEP $[23,24]$ and proposed several solutions such as antenna design [25] and interference management [26] to improve the performance of FDR-V2V systems. These works indicated that in the case of $\mathrm{V} 2 \mathrm{~V}$ communications, the channels between vehicles are not the traditional channels such as Rayleigh, Nakagami, and Rician. Instead, the double Rayleigh fading channels best describe the characteristics of V2V com- munication. Furthermore, under the impacts of both the RSI and V2V channels, the OP and SEP of FDR-V2V systems go to the error floor faster $[23,24]$.

On the other hand, when wireless devices move on the road and transmit signals for a long time, traditional power supply methods, such as wireline power supply, may be impossible to be used. Therefore, $\mathrm{EH}$ technique becomes an effective power supply for FDR-V2V communication systems. When equipped with $\mathrm{EH}$ circuits, the V2V devices can move and harvest the energy at the same time. Then, they use that harvested energy to transmit signals. However, due to the computational complexity caused by the double Raleigh fading channels, especially when $\mathrm{EH}$ is applied, the research about the EH-FDR-V2V system is still limited.

Motivated by these above matters, we mathematically evaluate the performance of an EH-FDR-V2V system over double Rayleigh fading channels. Although the combination of EH and FDR into a traditional wireless communication system has been investigated in various works, this is the first work that considers the combination of EH and FDR into a $\mathrm{V} 2 \mathrm{~V}$ communication system where the FD relay harvests energy from source and uses DF protocol. We successfully derive the exact expressions of the OP and throughput of the considered EH-FDR-V2V system. The main contributions of the paper can be summarized as follows:

(1) A model of the EH-FDR-V2V system is investigated where source is a static base station while relay and destination are moving vehicles. In addition, the relay harvests the energy from source via RF signals while moving on the road; thus, the channels from relay to destination are double Rayleigh fading channels. Moreover, the relay uses decode-and-forward (DF) protocol to forward data from source to destination

(2) The exact closed-form expressions of OP and throughput of the considered EH-FDR-V2V system under the impact of both RSI and double Rayleigh fading channels are obtained. We show that, compared with the Rayleigh fading channels, the double Rayleigh fading channels make the mathematical derivations more difficult

(3) The performance of the considered EH-FDR-V2V system is investigated in various scenarios. Numerical results show that the considered system is greatly reduced because of the double Rayleigh fading channels. With a certain transmission power of source, there is an optimal $\mathrm{EH}$ time duration which minimizes the OP and maximizes the throughput of the considered system. Furthermore, higher RSI makes the OP goes to outage floor faster. Therefore, based on the transmission power of source and the system requirements, we can choose a suitable value of the $\mathrm{EH}$ time duration to achieve both higher OP performance and throughput. Finally, we validate all analysis results by Monte-Carlo simulations

The rest of this paper is organized as follows: Section 2 describes the system and signal model of the considered 


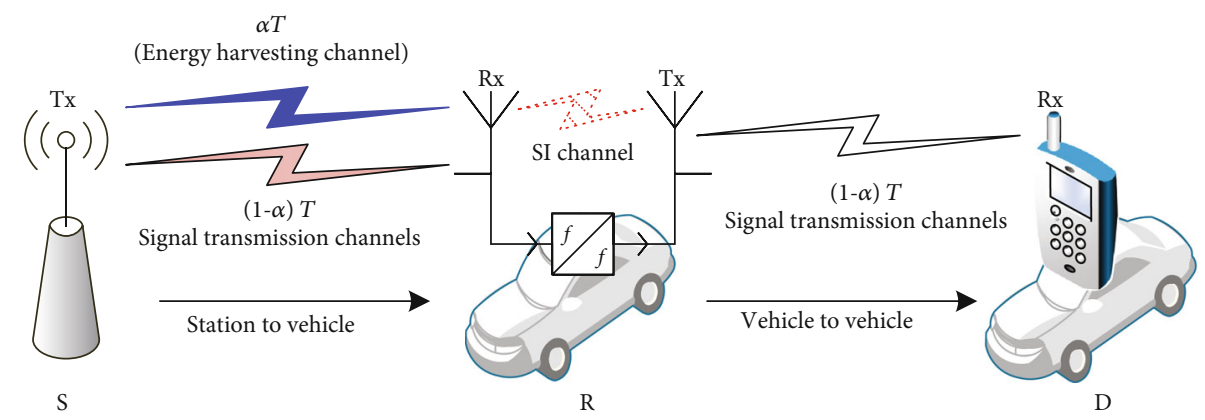

FIGURE 1: System and signal model of the considered EH-FDR-V2V system.

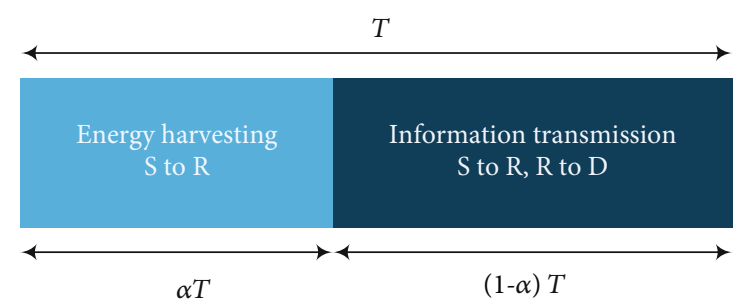

FIGURE 2: Operation of TS protocol at SWIPT receiver.

EH-FDR-V2V system. Then, Section 3 analyzes the performance of the considered system by deriving the exact closed-form expressions of OP and throughput. Section 4 presents the numerical results and discussions. Finally, Section 5 concludes the paper.

\section{System Models}

Figure 1 presents the system model of the considered EHFDR-V2V system. Data is transmitted from a static source (S) to a destination (D), a moving vehicle, via the assistance of relay $(\mathrm{R})$, also a moving vehicle. $S$ and $\mathrm{D}$ have one antenna and operate in HD mode while R has two antennas and operates in FD mode. In fact, $\mathrm{R}$ can use only one antenna for both transmitting and receiving, namely, shared antenna. However, the usage of separate antennas improves SIC capability because various methods for SIC in antenna domain such as isolation, antenna directionality, and cross-polarization can be easily applied [27]. Since the power supply of $\mathrm{R}$ is limited, especially when $\mathrm{R}$ moves on the road and exchanges data for a long time, $\mathrm{R}$ needs to harvest energy from the RF signals transmitted by $S$ and then uses all the harvested energy for signal transmission.

There are two EH protocols commonly used in wireless systems, i.e., TS and PS protocols as mentioned previously. In addition, the combination of these two protocols has also been applied in the literature to increase the amount of harvested energy and the performance of SWIPT systems. Various works such as [28-30] demonstrated that PS protocol can provide better secrecy outage probability (SOP) and ergodic secrecy rate than TS protocol. However, the throughput of TS protocol outperforms that of PS protocol at relatively low signal-to-noise-ratio (SNR) and high trans- mission rate [31]. Thus, we will use TS protocol for the analysis in this paper.

On the other hand, since $\mathrm{S}$ is stationary and $\mathrm{R}$ is a moving vehicle, the channel between $S$ and $R$ is influenced by Rayleigh fading because there is only one group of scatterers around the receiver $[32,33]$. Meanwhile, since both $\mathrm{R}$ and $\mathrm{D}$ are moving vehicles, the channel between $\mathrm{R}$ and $\mathrm{D}$ is affected by double (cascade) Rayleigh fading because there are two independent groups of scatterers around both the transmitter and receiver [32-37].

Figure 2 illustrates the operation of TS protocol at the SWIPT receiver in transmission block $T$. It comprises of two stages: $\mathrm{EH}$ and data transmission. In the first stage, the time duration of $\alpha T$ with time switching ratio $0 \leq \alpha \leq 1$ is used for R to harvest the energy from the RF signals transmitted from $\mathrm{S}$. In this stage, $\mathrm{S}$ can optimize the transmitted waveforms to enhance the harvested energy at R. Meanwhile, in the second stage, the time duration of $(1-\alpha) T$ is used for data transmission from $S$ to $R$ and from $R$ to $D$. Since $R$ simultaneously receives signals from $S$ and transmits them to $\mathrm{D}$ at the same time and on the same frequency band, the SI from transmission antenna to reception antenna of $\mathrm{R}$ occurs. With separate antenna for transmission and reception, R can have higher SIC capability compared with shared antennas. Furthermore, R can use both antennas for $\mathrm{EH}$ in the time duration $\alpha T$ to obtain higher the harvested energy. However, the work in [38] demonstrated that the usage of both antennas for $\mathrm{EH}$ will reduce the system performance compared with the case that only one antenna is used for EH. It is because higher harvested energy leads to higher transmission power of $\mathrm{R}$ and thus higher SI power. Consequently, in this paper, we consider the case that only one antenna of $\mathrm{R}$ is used for $\mathrm{EH}$.

In the time duration $\alpha \mathrm{T}$ for $\mathrm{EH}$, the harvested energy at $\mathrm{R}$ (denoted by $E_{h}^{\mathrm{R}}$ ) is given by [1]

$$
E_{h}^{\mathrm{R}}=\frac{\eta \alpha T P_{\mathrm{S}}\left|h_{\mathrm{SR}}\right|^{2}}{d_{\mathrm{SR}}^{m}},
$$

where $P_{\mathrm{S}}$ is the average transmission power of $\mathrm{S}$; $h_{\mathrm{SR}}$ is the fading coefficient of channel from $S$ to $\mathrm{R} ; d_{\mathrm{SR}}$ is the distance between $\mathrm{S}$ and $\mathrm{R} ; 2 \leq m \leq 6$ is the path loss exponent; and 0 $\leqslant \eta \leqslant 1$ is the energy conversion efficiency and its value is a constant but depends on the quality of electronic circuit. 
Because all the harvested energy is used for data transmission, the transmission power of $\mathrm{R}$ is computed as

$$
P_{\mathrm{R}}=\frac{\eta \alpha T P_{\mathrm{S}}\left|h_{\mathrm{SR}}\right|^{2}}{d_{\mathrm{SR}}^{m}(1-\alpha) T}=\frac{\eta \alpha P_{\mathrm{S}}\left|h_{\mathrm{SR}}\right|^{2}}{d_{\mathrm{SR}}^{m}(1-\alpha)} .
$$

In practice, the output power of $\mathrm{EH}$ circuit may be proportional to the harvested input power up to a certain saturation power threshold $P_{\text {th }}$. When the input power exceeds $P_{\text {th }}$, the output power remains unchanged. There are various factors causing the nonlinear characteristic of an energy harvester such as diode and saturation nonlinearities. Nonlinearities are an intrinsic property of diode. Meanwhile, saturation nonlinearities were confirmed by experiments on energy harvesting circuits [21,39-41]. As a result of this nonlinear characteristic, the transmission power of $\mathrm{R}$ with a nonlinear energy harvester can be obtained by extending (2) as

$$
P_{\mathrm{R}}=\left\{\begin{array}{l}
\frac{\eta \alpha P_{\mathrm{S}}\left|h_{\mathrm{SR}}\right|^{2}}{d_{\mathrm{SR}}^{m}(1-\alpha)}, \quad \frac{P_{\mathrm{S}}\left|h_{\mathrm{SR}}\right|^{2}}{d_{\mathrm{SR}}^{m}} \leq P_{\mathrm{th}} \\
\frac{\eta \alpha P_{\mathrm{th}}}{1-\alpha}, \quad \frac{P_{\mathrm{S}}\left|h_{\mathrm{SR}}\right|^{2}}{d_{\mathrm{SR}}^{m}}>P_{\mathrm{th}} .
\end{array}\right.
$$

In the time duration $(1-\alpha) T, \mathrm{R}$ receives signals from $\mathrm{S}$ while it forwards signals to D. This operation creates SI from the transmission antenna to the reception antenna of $\mathrm{R}$. The received signals at $\mathrm{R}$ and $\mathrm{D}$ are now expressed as

$$
\begin{aligned}
& y_{\mathrm{R}}=\frac{1}{\sqrt{d_{\mathrm{SR}}^{m}}} h_{\mathrm{SR}} \sqrt{P_{\mathrm{S}}} x_{\mathrm{S}}+\frac{1}{\sqrt{d_{\mathrm{RR}}^{m}}} \tilde{h}_{\mathrm{RR}} \sqrt{P_{\mathrm{R}}} x_{\mathrm{R}}+z_{\mathrm{R}}, \\
& y_{\mathrm{D}}=\frac{1}{\sqrt{d_{\mathrm{RD}}^{m}}} h_{\mathrm{RD}} \sqrt{P_{\mathrm{R}}} x_{\mathrm{R}}+z_{\mathrm{D}},
\end{aligned}
$$

where $d_{\mathrm{RR}}$ and $d_{\mathrm{RD}}$ are, respectively, the distances from the transmission to reception antennas of $\mathrm{R}$ and from $\mathrm{R}$ to $\mathrm{D}$; $h_{\mathrm{SR}}, h_{\mathrm{RD}}$, and $\tilde{h}_{\mathrm{RR}}$ are, respectively, the fading coefficients of $\mathrm{S}-\mathrm{R}$ channel and R-D channel and from the transmission antenna to the reception antenna of $\mathrm{R} ; x_{\mathrm{S}}$ and $x_{\mathrm{R}}$ are the transmitted signals at $\mathrm{S}$ and $\mathrm{R}$, respectively; and $P_{\mathrm{S}}$ and $P_{\mathrm{R}}$ are, respectively, the average transmission powers of $S$ and $\mathrm{R} ; z_{\mathrm{R}}$ and $z_{\mathrm{D}}$ are the Gaussian noises with zero mean and variance of $\sigma^{2}$, i.e., $z_{\mathrm{R}} \sim C \mathcal{N}\left(0, \sigma^{2}\right)$ and $z_{\mathrm{D}} \sim C \mathcal{N}\left(0, \sigma^{2}\right)$.

As shown in (4), the term $\left(1 / \sqrt{d_{\mathrm{RR}}^{m}}\right) \tilde{h}_{\mathrm{RR}} \sqrt{P_{\mathrm{R}}} x_{\mathrm{R}}$ is the SI. The average power of SI before SIC can be computed as

$$
E\left\{\frac{\left|\tilde{h}_{\mathrm{RR}}\right|^{2} P_{\mathrm{R}}}{d_{\mathrm{RR}}^{m}}\right\}=\frac{\eta \alpha P_{\mathrm{S}}}{1-\alpha} E\left\{\frac{\left|\tilde{h}_{\mathrm{RR}}\right|^{2}\left|h_{\mathrm{SR}}\right|^{2}}{d_{\mathrm{RR}}^{m}}\right\},
$$

where $E\{$.$\} is the expectation operator.$

Since R can apply all SIC techniques in three domains such as antenna propagation, analog suppression, and digital cancellation, the SI power is greatly reduced. Through SI channel estimation, SI can be suppressed effectively, especially in the digital cancellation domain. However, due to imperfect SIC, the residual SI (RSI) still exists at R. According to the analysis and measurements, the RSI due to FD mode (denoted by $I_{\mathrm{RSI}}$ ) is modeled by a complex Gaussian distribution with zero mean and variance of $\sigma_{\mathrm{RSI}}^{2}[3,8,9,42,43]$, where $\sigma_{\mathrm{RSI}}^{2}$ is given by

$$
\sigma_{\mathrm{RSI}}^{2}=\frac{k \eta \alpha P_{\mathrm{S}}}{1-\alpha}
$$

where $k$ denotes the SIC capability of the FD relay.

After all SIC techniques, (4) can be rewritten as

$$
y_{\mathrm{R}}=\frac{1}{\sqrt{d_{\mathrm{SR}}^{m}}} h_{\mathrm{SR}} \sqrt{P_{\mathrm{S}}} x_{\mathrm{S}}+I_{\mathrm{R}}+z_{\mathrm{R}} \text {. }
$$

Based on (8) and (5), the signal-to-interference-plusnoise ratios (SINRs) at R (denoted by $\gamma_{\mathrm{R}}$ ) and $\mathrm{D}$ (denoted by $\gamma_{\mathrm{D}}$ ) of the considered EH-FDR-V2V system are, respectively, computed as

$$
\begin{aligned}
& \gamma_{\mathrm{R}}=\frac{\left|h_{\mathrm{SR}}\right|^{2} P_{\mathrm{S}}}{d_{\mathrm{SR}}^{m}\left(\sigma_{\mathrm{RSI}}^{2}+\sigma^{2}\right)}, \\
& \gamma_{\mathrm{D}}=\frac{\left|h_{\mathrm{RD}}\right|^{2} P_{\mathrm{R}}}{d_{\mathrm{RD}}^{m} \sigma^{2}}=\frac{\left|h_{\mathrm{SR}}\right|^{2}\left|h_{\mathrm{RD}}\right|^{2} \eta \alpha P_{\mathrm{S}}}{d_{\mathrm{SR}}^{m} d_{\mathrm{RD}}^{m} \sigma^{2}(1-\alpha)} .
\end{aligned}
$$

When DF protocol is applied at FD relay, the end-to-end SINR (denoted by $\gamma$ ) of the considered system is calculated as

$$
\gamma=\min \left(\gamma_{\mathrm{R}}, \gamma_{\mathrm{D}}\right)
$$

\section{System Performance Analysis}

3.1. Outage Probability Analysis. In this subsection, we derive the analysis expression of the OP for evaluating the system performance. Mathematically, the $\mathrm{OP}$ is calculated as

$$
\begin{aligned}
\mathrm{OP} & =\operatorname{Pr}\left\{(1-\alpha) \log _{2}(1+\gamma)<\mathscr{R}\right\} \\
& =\operatorname{Pr}\left\{\gamma<2^{\mathscr{R} /(1-\alpha)}-1\right\} \\
& =\operatorname{Pr}\left\{\gamma<\gamma_{\text {th }}\right\},
\end{aligned}
$$

where $\mathscr{R}(\mathrm{bit} / \mathrm{s} / \mathrm{Hz})$ is a predata transmission rate; $\gamma$ is the end-to-end SINR of the considered EH-FDR-V2V system given in (10); and $\gamma_{\text {th }}=2^{\mathscr{R} /(1-\alpha)}-1$ is the SINR threshold.

From (11), the OP of the considered EH-FDR-V2V system is derived in Theorem 1 .

Theorem 1. Under the impact of the RSI, the OP of the considered EH-FDR-V2V system over double Rayleigh fading channels is given by 


$$
\begin{aligned}
O P= & 1-\frac{\pi}{2 N} \exp \left(-\frac{\Lambda \gamma_{t h}}{\Omega_{1}}\right) \sum_{n=1}^{N} \sqrt{\frac{\Theta \gamma_{t h}\left(1-\phi_{n}^{2}\right)}{\Omega_{2} \Omega_{3}\left(\Omega_{1} \ln (1 / u)+\Lambda \gamma_{t h}\right)}} \\
& \cdot K_{1}\left(\sqrt{\frac{\Theta \gamma_{t h}}{\Omega_{2} \Omega_{3}\left(\Omega_{1} \ln (1 / u)+\Lambda \gamma_{t h}\right)}}\right),
\end{aligned}
$$

where $\Lambda=\left(d_{S R}^{m}\left(\sigma_{R S I}^{2}+\sigma^{2}\right)\right) / P_{S} ; \Theta=\left(4 d_{S R}^{m} d_{R D}^{m} \sigma^{2}(1-\alpha)\right) / \eta \alpha$ $P_{S} ; \Omega_{1}$ is the average channel gain of Rayleigh fading $S-R$ channel; $\Omega_{2}$ and $\Omega_{3}$ are the average channel gains of the double Rayleigh $R-D$ channels; $N$ is the complexity-accuracy trade-off parameter; $\phi_{n}=\cos (((2 n-1) \pi) / 2 N) ; u=(1 / 2)$ $\left(\phi_{n}+1\right)$; and $K_{1}($.$) denotes the first-order modified Bessel$ function of the second kind [44].

Proof. Replacing $\gamma$ in (10) into (11), we have

$$
\mathrm{OP}=\operatorname{Pr}\left\{\min \left(\gamma_{\mathrm{R}}, \gamma_{\mathrm{D}}\right)<\gamma_{\mathrm{th}}\right\}=1-\operatorname{Pr}\left\{\gamma_{\mathrm{R}}>\gamma_{\mathrm{th}}, \gamma_{\mathrm{D}}>\gamma_{\mathrm{th}}\right\}
$$

Thus, it is obvious that the OP occurs when S-R or R-D link is in outage because $R$ is out of the coverage area of $S$ or $\mathrm{D}$ is out of the coverage area of $\mathrm{R}$.

Substituting $\gamma_{R}$ and $\gamma_{D}$ in (9) into (13), we rewrite (13) as

$$
\begin{aligned}
\mathrm{OP} & =1-\operatorname{Pr}\left\{\frac{\left|h_{\mathrm{SR}}\right|^{2} P_{\mathrm{S}}}{d_{\mathrm{SR}}^{m}\left(\sigma_{\mathrm{RSI}}^{2}+\sigma^{2}\right)}>\gamma_{\mathrm{th}}, \frac{\left|h_{\mathrm{SR}}\right|^{2}\left|h_{\mathrm{RD}}\right|^{2} \eta \alpha P_{\mathrm{S}}}{d_{\mathrm{SR}}^{m} d_{\mathrm{RD}}^{m} \sigma^{2}(1-\alpha)}>\gamma_{\mathrm{th}}\right\} \\
& =1-\operatorname{Pr}\left\{\left|h_{\mathrm{SR}}\right|^{2}>\frac{\gamma_{\mathrm{th}} d_{\mathrm{SR}}^{m}\left(\sigma_{\mathrm{RSI}}^{2}+\sigma^{2}\right)}{P_{\mathrm{S}}},\left|h_{\mathrm{RD}}\right|^{2}>\frac{\gamma_{\mathrm{th}} d_{\mathrm{SR}}^{m} d_{\mathrm{RD}}^{m} \sigma^{2}(1-\alpha)}{\left|h_{\mathrm{SR}}\right|^{2} \eta \alpha P_{\mathrm{S}}}\right\} \\
& =1-\left(1-\operatorname{Pr}\left\{\left|h_{\mathrm{RD}}\right|^{2} \leq\left.\frac{\gamma_{\mathrm{th}} d_{\mathrm{SR}}^{m} d_{\mathrm{RD}}^{m} \sigma^{2}(1-\alpha)}{\left|h_{\mathrm{SR}}\right|^{2} \eta \alpha P_{\mathrm{S}}}\right|_{\left|h_{\mathrm{SR}}\right|^{2}>\left(\left(\gamma_{\mathrm{th}} d_{\mathrm{SR}}^{m}\left(\sigma_{\mathrm{RSI}}^{2}+\sigma^{2}\right)\right) / P_{\mathrm{S}}\right)}\right\}\right) .
\end{aligned}
$$

Applying the property of conditional probability [45], (14) becomes

$$
\begin{aligned}
\mathrm{OP}= & 1-\int_{0}^{\infty}\left(1-F_{\left|h_{\mathrm{RD}}\right|^{2}}\left(\frac{\gamma_{\mathrm{th}} d_{\mathrm{SR}}^{m} d_{\mathrm{RD}}^{m} \sigma^{2}(1-\alpha)}{\left|h_{\mathrm{SR}}\right|^{2} \eta \alpha P_{\mathrm{S}}}\right)\right) \\
& \cdot f_{\left|h_{\mathrm{SR}}\right|^{2}}\left(y+\frac{\gamma_{\mathrm{th}} d_{\mathrm{SR}}^{m}\left(\sigma_{\mathrm{RSI}}^{2}+\sigma^{2}\right)}{P_{\mathrm{S}}}\right) d y
\end{aligned}
$$

where $y=\left|h_{\mathrm{SR}}\right|^{2}-\left(\left(\gamma_{\mathrm{th}} d_{\mathrm{SR}}^{m}\left(\sigma_{\mathrm{RSI}}^{2}+\sigma^{2}\right)\right) / P_{\mathrm{S}}\right)$.

For calculating the integral in (15), we need to derive the distributions of $\left|h_{\mathrm{SR}}\right|^{2}$ and $\left|h_{\mathrm{RD}}\right|^{2}$. Since S-R channel is influenced by Rayleigh fading, the cumulative distribution function (CDF, denoted by $F()$.$) and the probability density$ function (PDF, denoted by $f()$.$) of \left|h_{\mathrm{SR}}\right|^{2}$ are given by

$$
\begin{aligned}
& F_{\left|h_{\mathrm{SR}}\right|^{2}}(x)=1-\exp \left(-\frac{x}{\Omega_{1}}\right), \quad x \geqslant 0, \\
& f_{\left|h_{\mathrm{SR}}\right|^{2}}(x)=\frac{1}{\Omega_{1}} \exp \left(-\frac{x}{\Omega_{1}}\right), \quad x \geqslant 0,
\end{aligned}
$$

where $\Omega_{1}=E\left\{\left|h_{\mathrm{SR}}\right|^{2}\right\}$ is the average channel gain of S-R communication link.

Furthermore, since R-D channel is double Rayleigh fading channel, thus, $\left|h_{\mathrm{RD}}\right|^{2}$ is considered the multiplication of two independent variables $\left|h_{2}\right|^{2}$ and $\left|h_{3}\right|^{2}$, which means
$\left|h_{\mathrm{RD}}\right|^{2}=\left|h_{2}\right|^{2}\left|h_{3}\right|^{2}$ where $\left|h_{2}\right|^{2}$ and $\left|h_{3}\right|^{2}$ are the instantaneous channel gains of the Rayleigh fading channel with the average channel gains $\Omega_{2}=E\left\{\left|h_{2}\right|^{2}\right\}$ and $\Omega_{3}=E\left\{\left|h_{3}\right|^{2}\right\}$. The CDF and PDF of $\left|h_{\mathrm{RD}}\right|^{2}$ are, respectively, given by $[23,24,46]$

$$
F_{\left|h_{\mathrm{RD}}\right|^{2}}(x)=1-\sqrt{\frac{4 x}{\Omega_{2} \Omega_{3}}} K_{1}\left(\sqrt{\frac{4 x}{\Omega_{2} \Omega_{3}}}\right),
$$

$$
f_{\left|h_{\mathrm{RD}}\right|^{2}}(x)=\frac{2}{\Omega_{2} \Omega_{3}} K_{0}\left(\sqrt{\frac{4 x}{\Omega_{2} \Omega_{3}}}\right),
$$

where $K_{0}($.$) is the zero-order modified Bessel function of the$ second kind [44].

Now, applying (16), (17), (18), and (19) to calculate (15), we have

$$
\begin{aligned}
\mathrm{OP}= & 1-\int_{0}^{\infty} \sqrt{\frac{4 \gamma_{\mathrm{th}} d_{\mathrm{SR}}^{m} d_{\mathrm{RD}}^{m} \sigma^{2}(1-\alpha)}{\Omega_{2} \Omega_{3}\left(y+\left(\left(\gamma_{\mathrm{th}} d_{\mathrm{SR}}^{m}\left(\sigma_{\mathrm{RSI}}^{2}+\sigma^{2}\right)\right) / P_{\mathrm{S}}\right)\right) \eta \alpha P_{\mathrm{S}}}} K_{1} \\
& \cdot\left(\sqrt{\frac{4 \gamma_{\mathrm{th}} d_{\mathrm{SR}}^{m} d_{\mathrm{RD}}^{m} \sigma^{2}(1-\alpha)}{\Omega_{2} \Omega_{3}\left(y+\left(\left(\gamma_{\mathrm{th}} d_{\mathrm{SR}}^{m}\left(\sigma_{\mathrm{RSI}}^{2}+\sigma^{2}\right)\right) / P_{\mathrm{S}}\right)\right) \eta \alpha P_{\mathrm{S}}}}\right) \\
& \times \frac{1}{\Omega_{1}} \exp \left(-\frac{y}{\Omega_{1}}-\frac{\gamma_{\mathrm{th}} d_{\mathrm{SR}}^{m}\left(\sigma_{\mathrm{RSI}}^{2}+\sigma^{2}\right)}{\Omega_{1} P_{\mathrm{S}}}\right) d y
\end{aligned}
$$




$$
\begin{aligned}
= & 1-\frac{1}{\Omega_{1}} \int_{0}^{\infty} \sqrt{\frac{\Theta \gamma_{\mathrm{th}}}{\Omega_{2} \Omega_{3}\left(y+\Lambda \gamma_{\mathrm{th}}\right)}} K_{1} \\
& \cdot\left(\sqrt{\frac{\Theta \gamma_{\mathrm{th}}}{\Omega_{2} \Omega_{3}\left(y+\Lambda \gamma_{\mathrm{th}}\right)}}\right) \exp \left(-\frac{y}{\Omega_{1}}-\frac{\Lambda \gamma_{\mathrm{th}}}{\Omega_{1}}\right) d y \\
= & 1-\frac{1}{\Omega_{1}} \exp \left(-\frac{\Lambda \gamma_{\mathrm{th}}}{\Omega_{1}}\right) \int_{0}^{\infty} \sqrt{\frac{\Theta \gamma_{\mathrm{th}}}{\Omega_{2} \Omega_{3}\left(y+\Lambda \gamma_{\mathrm{th}}\right)}} K_{1} \\
& \cdot\left(\sqrt{\frac{\Theta \gamma_{\mathrm{th}}}{\Omega_{2} \Omega_{3}\left(y+\Lambda \gamma_{\mathrm{th}}\right)}}\right) \exp \left(-\frac{y}{\Omega_{1}}\right) d y .
\end{aligned}
$$

By changing the variable in (20), i.e., $z=\exp \left(-y / \Omega_{1}\right)$, (20) now becomes

$$
\begin{aligned}
\mathrm{OP}= & 1-\exp \left(-\frac{\Lambda \gamma_{\mathrm{th}}}{\Omega_{1}}\right) \int_{0}^{1} \sqrt{\frac{\Theta \gamma_{\mathrm{th}}}{\Omega_{2} \Omega_{3}\left(\Omega_{1} \ln (1 / z)+\Lambda \gamma_{\mathrm{th}}\right)}} K_{1} \\
& \cdot\left(\sqrt{\frac{\Theta \gamma_{\mathrm{th}}}{\Omega_{2} \Omega_{3}\left(\Omega_{1} \ln (1 / z)+\Lambda \gamma_{\mathrm{th}}\right)}}\right) d z .
\end{aligned}
$$

Using the Gaussian-Chebyshev quadrature method [47] for calculating the integral in (21), we have

$$
\begin{gathered}
\int_{0}^{1} \sqrt{\frac{\Theta \gamma_{\mathrm{th}}}{\Omega_{2} \Omega_{3}\left(\Omega_{1} \ln (1 / z)+\Lambda \gamma_{\mathrm{th}}\right)}} K_{1} \\
\left(\sqrt{\frac{\Theta \gamma_{\mathrm{th}}}{\Omega_{2} \Omega_{3}\left(\Omega_{1} \ln (1 / z)+\Lambda \gamma_{\mathrm{th}}\right)}}\right) d z \\
=\frac{\pi}{2 N} \sum_{n=1}^{N} \sqrt{\frac{\Theta \gamma_{\mathrm{th}}\left(1-\phi_{n}^{2}\right)}{\Omega_{2} \Omega_{3}\left(\Omega_{1} \ln (1 / u)+\Lambda \gamma_{\mathrm{th}}\right)}} K_{1} \\
\cdot\left(\sqrt{\frac{\Theta \gamma_{\mathrm{th}}}{\Omega_{2} \Omega_{3}\left(\Omega_{1} \ln (1 / u)+\Lambda \gamma_{\mathrm{th}}\right)}}\right)
\end{gathered}
$$

where $N, \phi_{n}$, and $u$ are defined after (12).

Plugging (22) into (21), we obtain the OP of the considered system as in (12). The proof is complete.

3.2. Throughput Analysis. For a wireless system, besides the $\mathrm{OP}$, throughput is another important parameter that needs to be evaluated. Therefore, in this subsection, we derive the throughput of the considered EH-FDR-V2V system.

Theorem 2. The throughput (denoted by $\mathscr{T}_{\text {put }}$ ) of the considered EH-FDR-V2V system is calculated as

$$
\mathscr{T}_{\text {put }}=\mathscr{R}(1-\alpha)(1-O P)
$$

where $\mathscr{R}$ and $\alpha$ are, respectively, the predata transmission rate and the time switching ratio and $O P$ is the outage probability, which is given in (12).

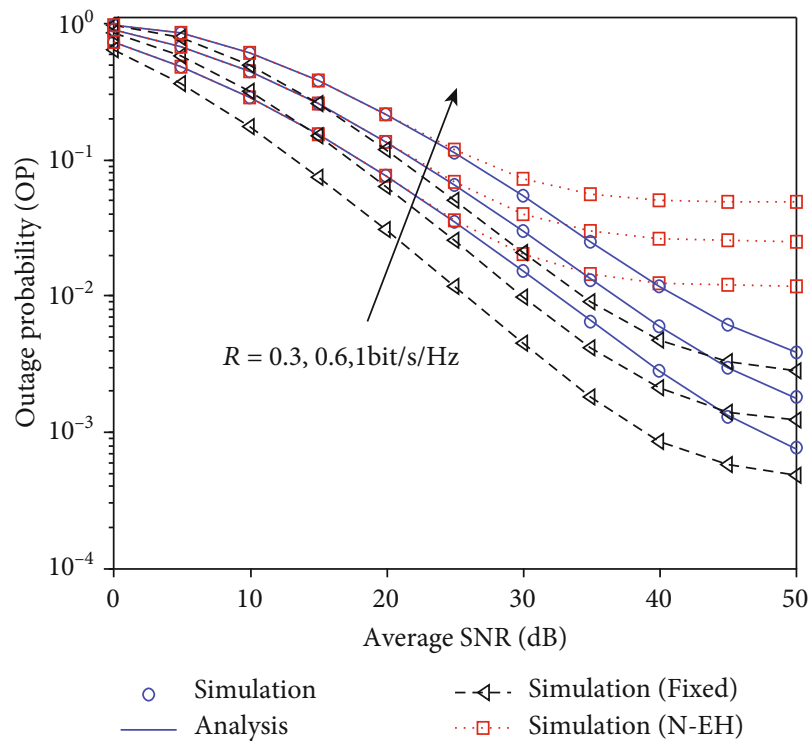

FIgURE 3: The impact of double Rayleigh fading channels on the OP of the considered EH-FDR-V2V system with $\mathscr{R}=0.3,0.6$, and $1 \mathrm{bit} / \mathrm{s} / \mathrm{Hz}, k=-30 \mathrm{~dB}$, and $\alpha=0.5$.

\section{Numerical Results and Discussion}

In this section, we use the OP and throughput expressions obtained in the previous section to evaluate the performance of the considered EH-FDR-V2V system. Various scenarios are carried out to investigate the impacts of the double Rayleigh fading channels, the RSI, and the time switching ratio on the system performance. The Monte-Carlo simulations are used to verify the correctness of our analysis. In all results, we set the average $\mathrm{SNR}=P_{\mathrm{S}} / \sigma^{2}$, the energy harvesting efficiency is $\eta=0.85$, the path loss exponent is $m=2.7$ (that is similar for an urban cellular network environment [31]), and the distances $\left(d_{\mathrm{SR}}\right.$ and $\left.d_{\mathrm{RD}}\right)$ and the average channel gains $\left(\Omega_{1}, \Omega_{2}\right.$, and $\left.\Omega_{3}\right)$ are normalized to a unit value such as in $[14,31,42]$.

Figure 3 plots the OP of the considered EH-FDR-V2V system versus the average SNR using (12) in Theorem 1. To evaluate the impact of double Rayleigh fading channels, we also provide the OP of this system in the case that $\mathrm{R}$ and $\mathrm{D}$ are static (denoted by "Fixed" in Figure 3). Note that in this case, S-R and R-D channels are influenced by Rayleigh fading. If $S$ and $D$ in [17] were equipped with a single antenna, the system model in [17] would become the case of "Simulation (Fixed)" in this paper. Moreover, we simulate the OP of the considered EH-FDR-V2V system with a nonlinear energy harvester and the saturation power threshold is $P_{\mathrm{th}} / \sigma^{2}=25 \mathrm{~dB}$ to compared with the OP of this system with a linear energy harvester. As can be seen from Figure 3, with $\mathscr{R}=0.3 \mathrm{bit} / \mathrm{s} / \mathrm{Hz}$ and $\mathrm{OP}=10^{-3}$, the OP performance of the considered EH-FDR-V2V system over double Rayleigh fading channels is $5 \mathrm{~dB}$ lower compared with that over Rayleigh fading channels. With a higher data transmission rate, e.g., $\mathscr{R}=0.6$ and $\mathscr{R}=1 \mathrm{bit} / \mathrm{s} / \mathrm{Hz}$, the OP in the case of double Rayleigh fading channels still goes down while the OP in the case of Rayleigh fading channels nearly goes to outage 


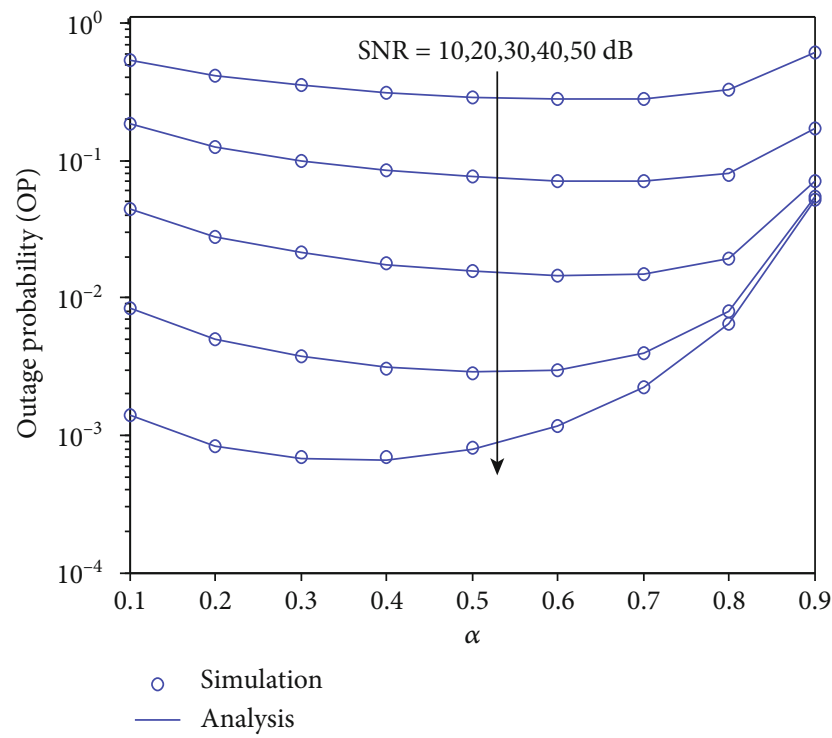

FIgure 4: The OP of the considered EH-FDR-V2V system versus the time switching ratio $\alpha$ with different values of SNR, $k=-30$ $\mathrm{dB}$, and $\mathscr{R}=0.3 \mathrm{bit} / \mathrm{s} / \mathrm{Hz}$.

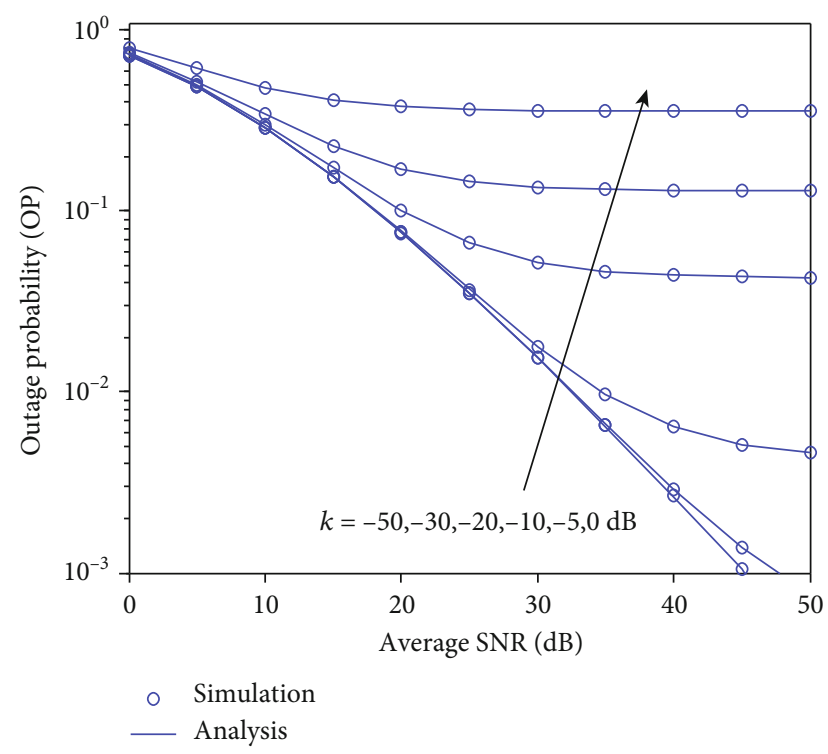

FIGURE 5: The impact of SIC capability on the OP of the considered EH-FDR-V2V system with $\mathscr{R}=0.3 \mathrm{bit} / \mathrm{s} / \mathrm{Hz}$ and $\alpha=0.5$.

floor due to the impact of the RSI. Furthermore, due to the effect of saturation power threshold, the OPs with nonlinear energy harvesters go to the floors faster than the OPs with linear energy harvesters as $\mathrm{SNR} \geq 35 \mathrm{~dB}$.

Figure 4 shows the OP of the considered EH-FDR-V2V system versus the time switching ratio $\alpha$ with different values of $\mathrm{SNR}$, i.e., $\mathrm{SNR}=10,20,30,40$, and $50 \mathrm{~dB}$. For a certain value of SNR, there is an optimal $\alpha$ that minimizes the OP of the considered system. For example, in the case of SNR = $50 \mathrm{~dB}$, the optimal $\alpha=0.3$. When SNR decreases, the optimal $\alpha$ is increased, i.e., $\alpha=0.5$ for $\mathrm{SNR}=40 \mathrm{~dB}, \alpha=0.6$ for $\mathrm{SNR}=30 \mathrm{~dB}$, and $\alpha=0.7$ for $\mathrm{SNR}=20,10 \mathrm{~dB}$. These results

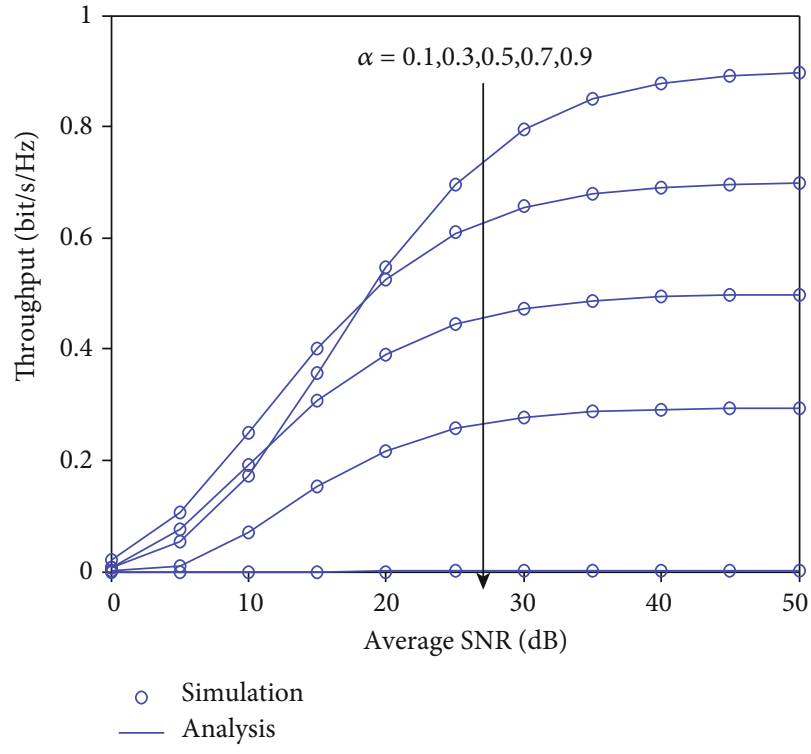

FIgURE 6: The throughput of the considered EH-FDR-V2V system versus the average SNR with different values of time switching ratio, $\mathscr{R}=1 \mathrm{bit} / \mathrm{s} / \mathrm{Hz}$, and $k=-30 \mathrm{~dB}$.

are reasonable for the considered system because it is obvious that, in low SNR regime (low transmission power of S), $R$ needs a long time to harvest enough energy for transmitting signals. In the case of high SNR regime, $R$ can harvest enough energy in a short time; thus, the optimal point is reduced. Based on these features, we can choose a suitable value of the time switching ratio to get the lowest $\mathrm{OP}$ of the considered system depending on the transmission power of $S$.

Figure 5 investigates the impact of SIC capability $k$ on the $\mathrm{OP}$ of the considered EH-FDR-V2V system. We can see in Figure 5 that with small values of $k$, e.g., $k=-50$ and $-30 \mathrm{~dB}$, the impact of the RSI on the OP is small. Therefore, the OP still goes down in the evaluated range of the SNR. However, with a higher value of $k$, e.g., $k=-20 \mathrm{~dB}$, the impact of the RSI is very strong, especially in the high SNR regime. Particularly, in the range from 0 to $30 \mathrm{~dB}$ of the SNR, the OPs with $k=-50,-30$, and $-20 \mathrm{~dB}$ are very similar. However, when SNR $>35 \mathrm{~dB}$, the differences between the OPs with $k=-50 \mathrm{~dB}, k=-30 \mathrm{~dB}$, and $k=-20 \mathrm{~dB}$ are remarkable. For $k=-20 \mathrm{~dB}$, the OP goes to outage floor when SNR $>50$ $\mathrm{dB}$. For a higher value of $k$, e.g., $k=-10,-5$, and $0 \mathrm{~dB}$, the OPs go to outage floor earlier (at $\mathrm{SNR}=35 \mathrm{~dB}$ ). The outage floors are $4 \times 10^{-2}, 1.3 \times 10^{-1}$, and $3.6 \times 10^{-1}$ corresponding to $k=-10 \mathrm{~dB}, k=-5 \mathrm{~dB}$, and $k=0 \mathrm{~dB}$, respectively. Therefore, all SIC solutions must be applied effectively to get the smallest RSI.

Figure 6 illustrates the throughput of the considered EHFDR-V2V system versus the average SNR for various time switching ratio $\alpha$. We use (23) in Theorem 2 to plot the analysis curves of the throughput. We show that lower $\alpha$ leads to higher throughput. It is because lower $\alpha$ means the duration time for $\mathrm{EH}$ is reduced; thus, the duration time for data exchange is increased. As a result, the considered system has higher throughput. With higher $\alpha$, the time duration for data exchange will be reduced, leading to a reduction in 


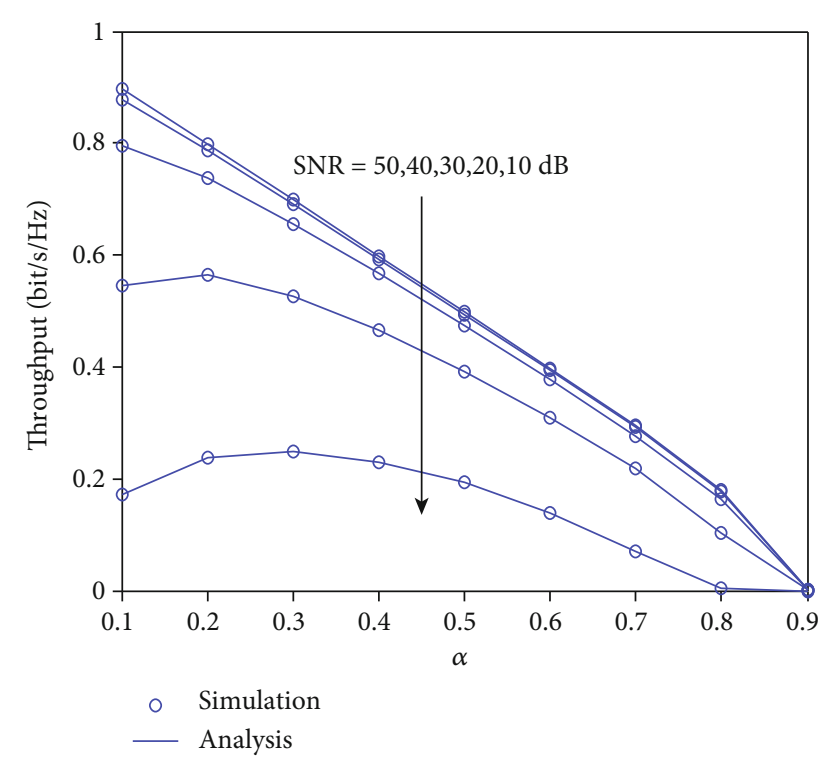

FIGURE 7: The throughput of the considered EH-FDR-V2V system versus the time switching ratio $\alpha$ with different values of SNR and $k=-30 \mathrm{~dB}$.

the throughput. It is also noted that, although we have higher throughput with lower $\alpha$, the usage of a small value of $\alpha$ can reduce the system performance because the destination (D) cannot detect its messages successfully. Therefore, we need to combine both the OP performance and the throughput to select a suitable $\alpha$. For the convenience in choosing $\alpha$ for the considered system, we will investigate the relationship between throughput and $\alpha$ in the next scenario.

Figure 7 investigates the throughput of the considered EH-FDR-V2V system versus the time switching ratio $\alpha$. We can see that, with high SNRs, the throughput is highest when the time switching ratio $\alpha$ is smallest. For example, in the case of $\mathrm{SNR}=50,40$, and $30 \mathrm{~dB}$, the throughput is maximal when $\alpha=0.1$. However, with lower SNRs, e.g., SNR $=20$ and $10 \mathrm{~dB}$, the value $\alpha=0.1$ is not the optimal value that maximizes the throughput. In these cases, the throughput is highest when $\alpha=0.2$ and $\alpha=0.3$ for $\mathrm{SNR}=20 \mathrm{~dB}$ and $\mathrm{SNR}=10 \mathrm{~dB}$, respectively. We should also remind that $\alpha=0$ should not be used to get the maximal throughput because when $\alpha=0$ (no time for $\mathrm{EH}$ ), the transmission power of R also equals zero. In this case, we have OP $=1$ and $\mathscr{T}_{\text {put }}=0$ (refer to (23)). Based on Figures 4 and 7, depending on the requirements of OP and throughput, we can choose a suitable value of $\alpha$ for the considered EH-FDR-V2V system. For example, in the case of $\mathrm{SNR}=50 \mathrm{~dB}$, we can choose the range of $\alpha$ from 0.1 to 0.3 to get high OP performance and throughput.

\section{Conclusions}

Motivated by the big advantages of the $\mathrm{EH}$ technique, in this paper, we investigated the performance of the EH-FDR-V2V system under the impact of RSI due to the FD transmission mode over double Rayleigh fading channels. We successfully derived the closed-form expressions of the outage probability and throughput of the considered system. Based on these expressions, the system performance is investigated through various scenarios. Numerical results showed that the performance of the considered system is greatly reduced compared with that system over Rayleigh fading channels. With a certain value of the transmission power of source, there is an optimal EH time duration that minimizes the OP and maximizes the throughput. Therefore, based on the transmission power of source and the system requirements, we can choose a suitable $\mathrm{EH}$ time duration to get high OP performance and throughput. Furthermore, the RSI causes the outage floor in the considered system, especially in the case of high RSI. Thus, effective SIC techniques should be used when deploying the FD systems in practice.

\section{Data Availability}

The data used to support the findings of this study are available from the corresponding author upon request.

\section{Conflicts of Interest}

The authors declare that they have no conflicts of interest.

\section{References}

[1] X. Lu, P. Wang, D. Niyato, D. I. Kim, and Z. Han, "Wireless networks with RF energy harvesting: a contemporary survey," IEEE Communications Surveys \& Tutorials, vol. 17, no. 2, pp. 757-789, 2015.

[2] V.-D. Nguyen, T. Q. Duong, H. D. Tuan, O.-S. Shin, and H. V. Poor, "Spectral and energy efficiencies in full-duplex wireless information and power transfer," IEEE Transactions on Communications, vol. 65, no. 5, pp. 2220-2233, 2017.

[3] B. C. Nguyen, T. M. Hoang, P. T. Tran, and T. N. Nguyen, "Outage probability of NOMA system with wireless power transfer at source and full-duplex relay," AEU-International Journal of Electronics and Communications, vol. 116, article 152957, 2020.

[4] H. H. M. Tam, H. D. Tuan, A. A. Nasir, T. Q. Duong, and H. V. Poor, "MIMO energy harvesting in full-duplex multi-user networks," IEEE Transactions on Wireless Communications, vol. 16, no. 5, pp. 3282-3297, 2017.

[5] Y. Deng, K. J. Kim, T. Q. Duong, M. Elkashlan, G. K. Karagiannidis, and A. Nallanathan, "Full-duplex spectrum sharing in cooperative single carrier systems," IEEE Transactions on Cognitive Communications and Networking, vol. 2, no. 1, pp. 6882, 2016.

[6] X.-T. Doan, N.-P. Nguyen, C. Yin, D. B. Da Costa, and T. Q. Duong, "Cognitive full-duplex relay networks under the peak interference power constraint of multiple primary users," EURASIP Journal on Wireless Communications and Networking, vol. 2017, no. 1, Article ID 8, 2017.

[7] B. C. Nguyen, X. N. Tran, and D. T. Tran, "Performance analysis of in-band full-duplex amplify-and-forward relay system with direct link," in 2018 2nd International Conference on Recent Advances in Signal Processing, Telecommunications \& Computing (SigTelCom), pp. 192-197, Ho Chi Minh City, Vietnam, January 2018.

[8] B. C. Nguyen, X. N. Tran, D. T. Tran, and L. T. Dung, "Fullduplex amplify-and-forward relay system with direct link: 
performance analysis and optimization," Physical Communication, vol. 37, article 100888, 2019.

[9] D. Bharadia, E. McMilin, and S. Katti, "Full duplex radios," ACM SIGCOMM Computer Communication Review, vol. 43, no. 4, pp. 375-386, 2013.

[10] A. H. Gazestani, S. A. Ghorashi, B. Mousavinasab, and M. Shikh-Bahaei, "A survey on implementation and applications of full duplex wireless communications," Physical Communication, vol. 34, pp. 121-134, 2019.

[11] B. C. Nguyen and X. N. Tran, "Performance analysis of full-duplex amplify-and-forward relay system with hardware impairments and imperfect self-interference cancellation," Wireless Communications and Mobile Computing, vol. 2019, Article ID 4946298, 10 pages, 2019.

[12] B. C. Nguyen, T. M. Hoang, and P. T. Tran, "Performance analysis of full-duplex decode-and-forward relay system with energy harvesting over Nakagami- $m$ fading channels," AEUInternational Journal of Electronics and Communications, vol. 98, pp. 114-122, 2019.

[13] I. Krikidis, H. A. Suraweera, P. J. Smith, and C. Yuen, "Fullduplex relay selection for amplify-and-forward cooperative networks," IEEE Transactions on Wireless Communications, vol. 11, no. 12, pp. 4381-4393, 2012.

[14] B. C. Nguyen, N. N. Thang, X. N. Tran, and L. T. Dung, "Impacts of imperfect channel state information, transceiver hardware, and self-interference cancellation on the performance of full-duplex mimo relay system," Sensors, vol. 20, no. 6, article 1671, 2020.

[15] B. C. Nguyen, T. M. Hoang, S.-G. Choi, and L. T. Dung, "Fullduplex relay system with energy harvesting: outage and symbol error probabilities," in 2018 International Conference on Advanced Technologies for Communications (ATC), pp. 360365, Ho Chi Minh City, Vietnam, October 2018.

[16] Y. Alsaba, C. Y. Leow, and S. K. A. Rahim, "Full-duplex cooperative non-orthogonal multiple access with beamforming and energy harvesting," IEEE Access, vol. 6, pp. 19726-19738, 2018.

[17] T. M. Hoang, N. T. Tan, N. B. Cao, and L. T. Dung, "Outage probability of MIMO relaying full-duplex system with wireless information and power transfer," in 2017 Conference on Information and Communication Technology (CICT), pp. 1-6, Gwalior, India, November 2017.

[18] B. C. Nguyen, T. M. Hoang, X. N. Pham, and P. T. Tran, "Performance analysis of energy harvesting-based full-duplex decode-and-forward vehicle-to-vehicle relay networks with nonorthogonal multiple access," Wireless Communications and Mobile Computing, vol. 2019, Article ID 6097686, 11 pages, 2019.

[19] G. Nauryzbayev, M. Abdallah, and K. M. Rabie, "Outage probability of the EH-based full-duplex AF and DF relaying systems in $\alpha-\mu$ environment," in 2018 IEEE 88th Vehicular Technology Conference (VTC-Fall), pp. 1-6, Chicago, IL, USA, August 2018.

[20] X. Zhou, R. Zhang, and C. K. Ho, "Wireless information and power transfer: architecture design and rate-energy tradeoff," IEEE Transactions on Communications, vol. 61, no. 11, pp. 4754-4767, 2013.

[21] B. Clerckx, R. Zhang, R. Schober, D. W. K. Ng, D. I. Kim, and H. V. Poor, "Fundamentals of wireless information and power transfer: from RF energy harvester models to signal and system designs," IEEE Journal on Selected Areas in Communications, vol. 37, no. 1, pp. 4-33, 2019.
[22] C. Campolo, A. Molinaro, A. O. Berthet, and A. Vinel, "Fullduplex radios for vehicular communications," IEEE Communications Magazine, vol. 55, no. 6, pp. 182-189, 2017.

[23] B. C. Nguyen, X. N. Tran, T. M. Hoang, and L. T. Dung, "Performance analysis of full-duplex vehicle-to-vehicle relay system over double-rayleigh fading channels," Mobile Networks and Applications, vol. 25, no. 1, pp. 363-372, 2020.

[24] B. C. Nguyen, T. M. Hoang, and L. T. Dung, "Performance analysis of vehicle-to-vehicle communication with fullduplex amplify-and-forward relay over double-rayleigh fading channels," Vehicular Communications, vol. 19, p. 100166 , 2019.

[25] C.-X. Mao, S. Gao, and Y. Wang, "Dual-band full-duplex $\mathrm{Tx} / \mathrm{Rx}$ antennas for vehicular communications," IEEE Transactions on Vehicular Technology, vol. 67, no. 5, pp. 40594070, 2018.

[26] M. Yang, S.-W. Jeon, and D. K. Kim, "Interference management for in-band full-duplex vehicular access networks," IEEE Transactions on Vehicular Technology, vol. 67, no. 2, pp. 18201824, 2018.

[27] A. Sabharwal, P. Schniter, D. Guo, D. W. Bliss, S. Rangarajan, and R. Wichman, "In-band full-duplex wireless: challenges and opportunities," IEEE Journal on Selected Areas in Communications, vol. 32, no. 9, pp. 1637-1652, 2014.

[28] S. Sharma, S. D. Roy, and S. Kundu, "Secrecy outage in a twohop decode and forward relay network with accumulated harvested energy," Physical Communication, vol. 36, article 100792, 2019.

[29] S. Sharma, S. D. Roy, and S. Kundu, "Secure communication with energy harvesting multiple half-duplex DF relays assisted with jamming," Wireless Networks, vol. 26, pp. 1151-1164, 2020.

[30] S. Sharma, S. Dhar Roy, and S. Kundu, "Secure communication in cognitive radio networks with untrusted AF relays," International Journal of Communication Systems, vol. 32, no. 7, article e3919, 2019.

[31] A. A. Nasir, X. Zhou, S. Durrani, and R. A. Kennedy, "Relaying protocols for wireless energy harvesting and information processing," IEEE Transactionson Wireless Communications, vol. 12, no. 7, pp. 3622-3636, 2013.

[32] I. Z. Kovacs, P. C. F. Eggers, K. Olesen, and L. G. Petersen, "Investigations of outdoor-to-indoor mobile-to-mobile radio communication channels," in Proceedings IEEE 56th Vehicular Technology Conference, vol. 1, pp. 430-434, Vancouver, BC, Canada, 2002.

[33] J. Salo, H. M. El-Sallabi, and P. Vainikainen, "Statistical analysis of the multiple scattering radio channel," IEEE Transactions on Antennas and Propagation, vol. 54, no. 11, pp. 3114-3124, 2006.

[34] J. Salo, H. M. El-Sallabi, and P. Vainikainen, "Impact of double-Rayleigh fading on system performance," in 2006 1st International Symposium on Wireless Pervasive Computing, pp. 1-5, Phuket, Thailand, 2006.

[35] T. T. Duy, G. C. Alexandropoulos, V. T. Tung, V. N. Son, and T. Q. Duong, "Outage performance of cognitive cooperative networks with relay selection over double-Rayleigh fading channels," IET Communications, vol. 10, no. 1, pp. 57-64, 2016.

[36] A. S. Akki and F. Haber, "A statistical model of mobile-tomobile land communication channel," IEEE Transactions on Vehicular Technology, vol. 35, no. 1, pp. 2-7, 1986. 
[37] I. Kovacs, Radio Channel Characterisation for Private Mobile Radio Systems: Mobile-to-Mobile Radio Link Investigations, [Ph.D. Thesis], Aalborg Universitet, 2002.

[38] C. Zhong, H. A. Suraweera, G. Zheng, I. Krikidis, and Z. Zhang, "Wireless information and power transfer with full duplex relaying," IEEE Transactions on Communications, vol. 62, no. 10, pp. 3447-3461, 2014.

[39] Y. Dong, M. J. Hossain, and J. Cheng, "Performance of wireless powered amplify and forward relaying over Nakagami- fading channels with nonlinear energy harvester," IEEE Communications Letters, vol. 20, no. 4, pp. 672-675, 2016.

[40] Z. Wei, S. Sun, X. Zhu, D. I. Kim, and D. W. K. Ng, "Resource allocation for wireless-powered full-duplex relaying systems with nonlinear energy harvesting efficiency," IEEE Transactions on Vehicular Technology, vol. 68, no. 12, pp. 1207912093, 2019.

[41] K. Xu, M. Zhang, J. Liu, N. Sha, W. Xie, and L. Chen, "SWIPT in mMIMO system with non-linear energy-harvesting terminals: protocol design and performance optimization," EURASIP Journal on Wireless Communications and Networking, vol. 2019, no. 1, Article ID 72, 2019.

[42] C. Li, Z. Chen, Y. Wang, Y. Yao, and B. Xia, "Outage analysis of the full-duplex decode-and-forward two-way relay system," IEEE Transactions on Vehicular Technology, vol. 66, no. 5, pp. 4073-4086, 2017.

[43] X. N. Tran, B. C. Nguyen, and D. T. Tran, "Outage probability of two-way full-duplex relay system with hardware impairments," in 2019 3rd International Conference on Recent Advances in Signal Processing, Telecommunications \& Computing (SigTelCom), pp. 135-139, Hanoi, Vietnam, 2019.

[44] A. Jeffrey and D. Zwillinger, Table of Integrals, Series, and Products, Academic Press, 2007.

[45] A. Leon-Garcia and A. Leon-Garcia, Probability, Statistics, and Random Processes for Electrical Engineering, Pearson/Prentice Hall, Upper Saddle River, NJ, USA, 3rd edition, 2008.

[46] Y. Ai, M. Cheffena, A. Mathur, and H. Lei, "On Physical Layer Security of Double Rayleigh Fading Channels for Vehicular Communications," IEEE Wireless Communications Letters, vol. 7, no. 6, pp. 1038-1041, 2018.

[47] F. B. Hildebrand, Introduction to Numerical Analysis, Courier Corporation, 1987. 